\title{
DMD-encoded spectral photoacoustic microscopy
}

\section{Yu Wang, Konstantin Maslov, Lihong V. Wang}

Yu Wang, Konstantin Maslov, Lihong V. Wang, "DMD-encoded spectral photoacoustic microscopy," Proc. SPIE 8223, Photons Plus Ultrasound: Imaging and Sensing 2012, 822312 (23 February 2012); doi: $10.1117 / 12.910521$

SPIE. Event: SPIE BiOS, 2012, San Francisco, California, United States 


\title{
DMD-encoded spectral photoacoustic microscopy
}

\author{
Yu Wang, Konstantin Maslov, Lihong V. Wang* \\ Optical Imaging Laboratory, Dept. of Biomedical Engineering, Washington University in St. Louis, \\ 1 Brookings Dr., St. Louis, MO, USA 63130
}

\begin{abstract}
We present a spectrally encoded photoacoustic microscope based on a digital mirror device (DMD). It enables fast spatially resolved spectral measurements of optical absorption. The imaging system can quickly tune the laser illumination spectrum at the laser pulse repetition rate of $2 \mathrm{kHz}$. To demonstrate multi-wavelength absorption measurements, we imaged optically absorbing solution phantom. Compared with spectral scanning, spectral encoding recovers chromophore absorption spectra with improved accuracy by enhancing photoacoustic amplitude signal-to-noise ratio.
\end{abstract}

Keywords: Photoacoustic microscopy, spectral encoding, digital mirror device, photoacoustic spectroscopy

\section{INTRODUCTION}

Multiwavelength photoacoustic (PA) imaging provides in vivo label-free measurement of the optical absorption of fluorescent or non-fluorescent molecules [1,2]. By tuning the laser illumination wavelength, i.e. spectral scanning, optical absorption spectra is acquired by PA amplitude. The current laser systems, such as the dye laser, Ti-sapphire laser or OPO laser, make it inherently difficult to tune the wavelength quickly enough for PA imaging [3-5]. Fast wavelength tuning can reduce the time it takes to acquire a stack of multi-wavelength PA images so that spectraresolvable dynamic processes can be imaged. Moreover, it can mitigate the motion artifacts for in vivo PA imaging.

To speed up multiwavelength PA imaging, we developed spectrally encoded PA microscopy based on a digital mirror device (DMD) [6]. The system utilizes a DMD to multiplex the illumination wavelengths from a broadband light source, a laser pumped dye cell. By switching the wavelength at the laser pulse repetition rate, it is capable of fast quantitative spectroscopic measurement. Moreover, with Hadamard spectra encoding, the absorption spectra can be recovered with improved accuracy. When signal to noise ratio (SNR) is low, the spectral encoding strategy of absorption measurement can take full advantage of the wide illumination spectrum. Thus the PA imaging system can be made compact and inexpensive with a laser pumped dye cell, a photonic crystal fiber laser [7] and a laser diode array [8].

\section{METHOD}

\subsection{Spectral PA microscope based on a DMD}

A schematic of the spectrally encoded PA imaging system is shown in Fig. 1. A Nd:YLF laser (INNOSLAB, Edgewave) pumped laser dye cell provides broadband illumination [9]. The circulating dye cell contains a mixture of Rhodamine 6G and Rhodamine B ethanol solution as a laser dye solution to extend the lasing wavelength range. A broadband laser

*1hwang@wustl.edu;

Photons Plus Ultrasound: Imaging and Sensing 2012, edited by Alexander A. Oraevsky, Lihong V. Wang, Proc. of SPIE Vol. 8223, 822312 · (C) 2012 SPIE · CCC code: 1605-7422/12/\$18 - doi: 10.1117/12.910521 
beam with an emission wavelength range from $570 \mathrm{~nm}$ to $590 \mathrm{~nm}$ emerges from the ends of the dye cell. The laser pulse duration is $7 \mathrm{~ns}$. The laser pulse repetition, the data acquisition and the $\mathrm{x}-\mathrm{y}$ scanning are synchronized at $\sim 2 \mathrm{kHz}$. The collimated light beam is dispersed through a transmission diffraction grating (600 lines $/ \mathrm{mm}$ blazed at $560 \mathrm{~nm}$, Wasatch Photonics) and imaged onto the surface of the digital mirror device (DLP Discovery 4100, Texas Instruments) with a cylindrical lens of $300 \mathrm{~mm}$ focal length. The broadband illumination has a line dispersion of $5.5 \mathrm{~nm} / \mathrm{mm}$ on the DMD surface. The digital mirror device has $1024 \times 768$ microscopic mirrors which can be individually tilted $\pm 12^{\circ}$, to an on or off state. Each column of the DMD mirrors corresponds to a different wavelength. By changing the mirror pattern, the illumination wavelengths are selected. The laser light of selected wavelengths is spatially recombined by another set of cylindrical lens and diffraction grating, and then is directed to the multi-wavelength PA imaging system. The broadband emission spectra are encoded at the laser pulse repetition rate. Thus multi-wavelength PA measurement is performed at 2 $\mathrm{kHz}$. The PA imaging system consists of a microscopic objective and an ultrasonic transducer [10,11], which are confocally and co-axially aligned. Two-dimensional raster scanning of the imaging object constructs a three-dimensional image of optical absorption within the tissue.

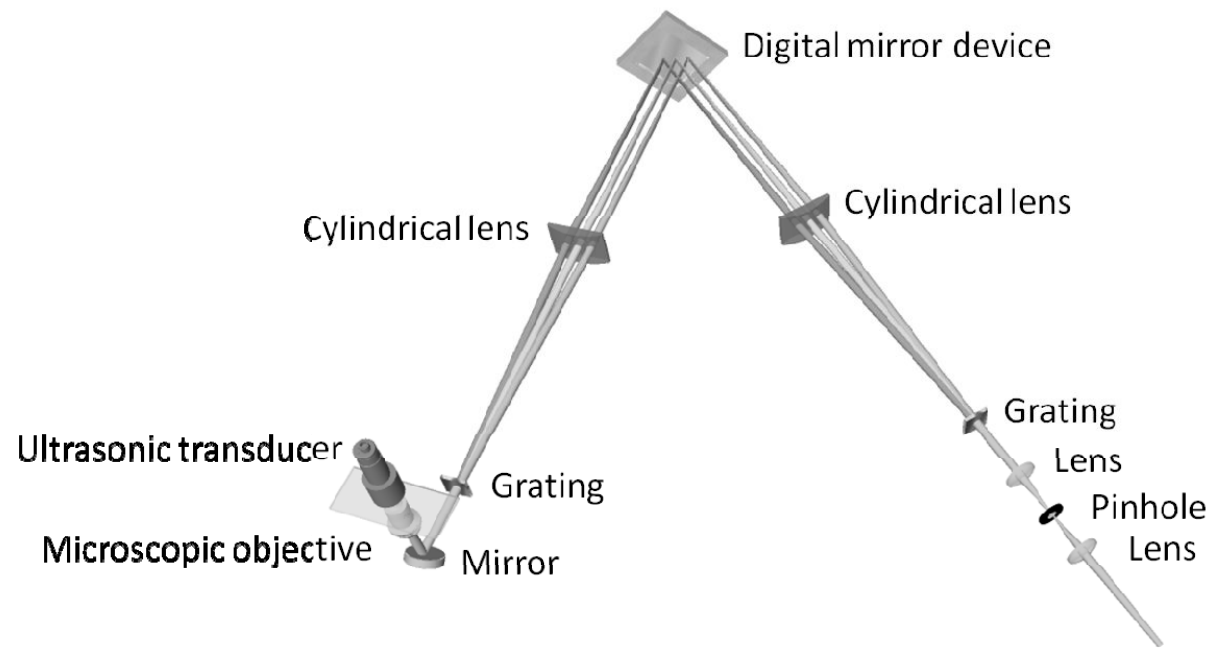

Fig. 1. Schematic of the spectral encoding PA imaging system.

\subsection{Spectrally encoded PA measurements}

We used a set of one-dimensional (1D) Hadamard sequences as the wavelength multiplexing pattern in the same way as in Hadamard spectroscopy. Instead of irradiating the sample with a light beam at a single wavelength, many wavelengths can be selected at the same time using the digital mirror device. If a broadband light source of constant output pulse energy is used, the signal-to-noise ratio for multi-wavelength photoacoustic imaging can be improved by encoding the optical illumination spectrum in comparison to filtering for one wavelength at a time. The 2D DMD encodes the 1D Hadamard imaging pattern by alternating mirrors' on and off states in a column-wise manner. A set of 15-element Hadamard sequences were used to encode the 15 wavelengths. The DMD sequentially loaded the 15 Hadamard encoding pattern and recorded the corresponding PA signal amplitude at each laser pulse. A stack of spectrally encoded PA images was acquired to recover the optical absorption spectrum [12,13]. The spectrally encoded PA absorption measurements can be described by a linearly independent set of equations (Eq. 1). The encoded spectra information was then transformed into the absorption spectra using matrix inversion.

$$
P_{H}=H_{15} \cdot P_{I}
$$


where $P_{I}$ is the PA amplitude at 15 individual spectral elements; $P_{H}$ is the PA amplitude generated with 15 Hadamard masks; and $H_{15}$ is the Hadamard encoding matrix. The multi-wavelength PA images were acquired at 15 wavelengths, from $574 \mathrm{~nm}$ to $586 \mathrm{~nm}$. The multi-wavelength measurements can be recovered by the inverse transformation. Meanwhile, spectral scanning PA measurement was performed by switching across the 15 individual wavelengths.

The Hadamard transform matrix of order 15 is

$$
H=\left(\begin{array}{lllllllllllllll}
1 & 0 & 1 & 0 & 1 & 0 & 1 & 0 & 1 & 0 & 1 & 0 & 1 & 0 & 1 \\
0 & 1 & 1 & 0 & 0 & 1 & 1 & 0 & 0 & 1 & 1 & 0 & 0 & 1 & 1 \\
1 & 1 & 0 & 0 & 1 & 1 & 0 & 0 & 1 & 1 & 0 & 0 & 1 & 1 & 0 \\
0 & 0 & 0 & 1 & 1 & 1 & 1 & 0 & 0 & 0 & 0 & 1 & 1 & 1 & 1 \\
1 & 0 & 1 & 1 & 0 & 1 & 0 & 0 & 1 & 0 & 1 & 1 & 0 & 1 & 0 \\
0 & 1 & 1 & 1 & 1 & 0 & 0 & 0 & 0 & 1 & 1 & 1 & 1 & 0 & 0 \\
1 & 1 & 0 & 1 & 0 & 0 & 1 & 0 & 1 & 1 & 0 & 1 & 0 & 0 & 1 \\
0 & 0 & 0 & 0 & 0 & 0 & 0 & 1 & 1 & 1 & 1 & 1 & 1 & 1 & 1 \\
1 & 0 & 1 & 0 & 1 & 0 & 1 & 1 & 0 & 1 & 0 & 1 & 0 & 1 & 0 \\
0 & 1 & 1 & 0 & 0 & 1 & 1 & 1 & 1 & 0 & 0 & 1 & 1 & 0 & 0 \\
1 & 1 & 0 & 0 & 1 & 1 & 0 & 1 & 0 & 0 & 1 & 1 & 0 & 0 & 1 \\
0 & 0 & 0 & 1 & 1 & 1 & 1 & 1 & 1 & 1 & 1 & 0 & 0 & 0 & 0 \\
1 & 0 & 1 & 1 & 0 & 1 & 0 & 1 & 0 & 1 & 0 & 0 & 1 & 0 & 1 \\
0 & 1 & 1 & 1 & 1 & 0 & 0 & 1 & 1 & 0 & 0 & 0 & 0 & 1 & 1 \\
1 & 1 & 0 & 1 & 0 & 0 & 1 & 1 & 0 & 0 & 1 & 0 & 1 & 1 & 0
\end{array}\right)
$$

\section{RESULTS}

\subsection{Phantom measurement}

To demonstrate the spectrally encoded PA imaging system's capability of spectroscopic measurement, a red ink aqueous solution of five-fold dilution was imaged. The laser pulse energy is $\sim 3 \mathrm{~nJ}$ at $586 \mathrm{~nm}$. The accuracy of the spectral scanning and of the spectrally encoded PA measurement of absorption spectra was compared on the basis of the percent error [7]. Figure 2 shows the PA A-line signals acquired with spectral scanning and spectrally encoding methods. Because of a low SNR, the transient PA peak signal cannot be extracted from the noise. However, the spectrally encoded PA A-line signal has increased SNR by delivering eight wavelengths simultaneously. 


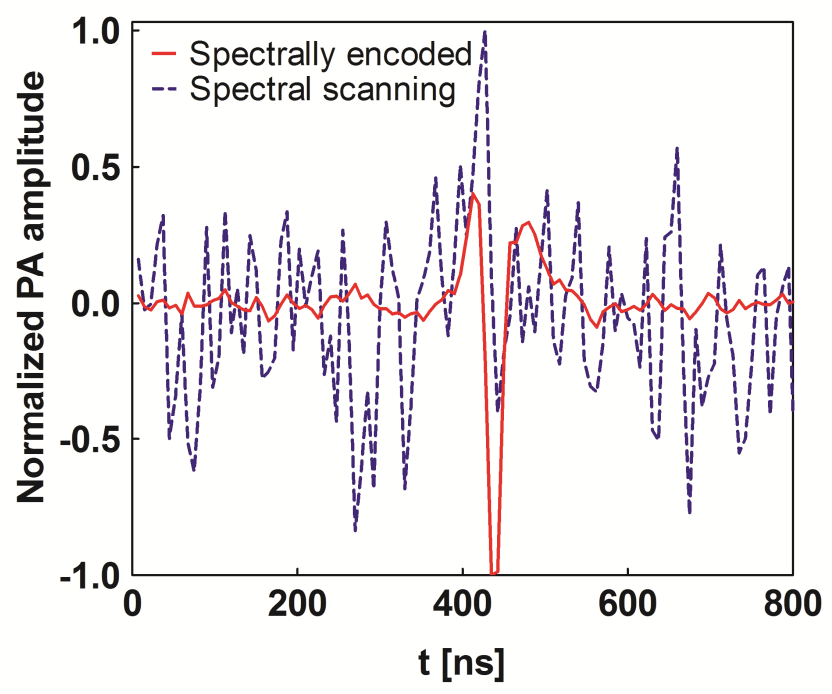

Fig. 2. PA A-line signals acquired using spectral scanning and spectrally encoded PA measurements.

Unlike traditional optical imaging modalities, the PA imaging acquires a time of flight signal that has depth profiling capabilities. The peak amplitudes of PA signals can be attributed to different internal structures. To extract the optical absorption coefficients, the maximum peak-to-peak amplitudes is used. In Fig. 3, the optical absorption coefficients measured by spectral scanning are compared with those recovered by spectrally encoded PA measurements. The spectrally encoded PA measurements recovered the absorption coefficients over the range of wavelengths from $574 \mathrm{~nm}$ to $586 \mathrm{~nm}$, with less than $3 \%$ error. However, the spectral scanning PA measurement showed $>16 \%$ error. The error increases above $583 \mathrm{~nm}$ and below $577 \mathrm{~nm}$ where the laser pulse energy was relatively low. This is explained by the low PA signal SNR. The mathematical model of the maximum peak to peak amplitude was not proportional to the optical absorption coefficients.

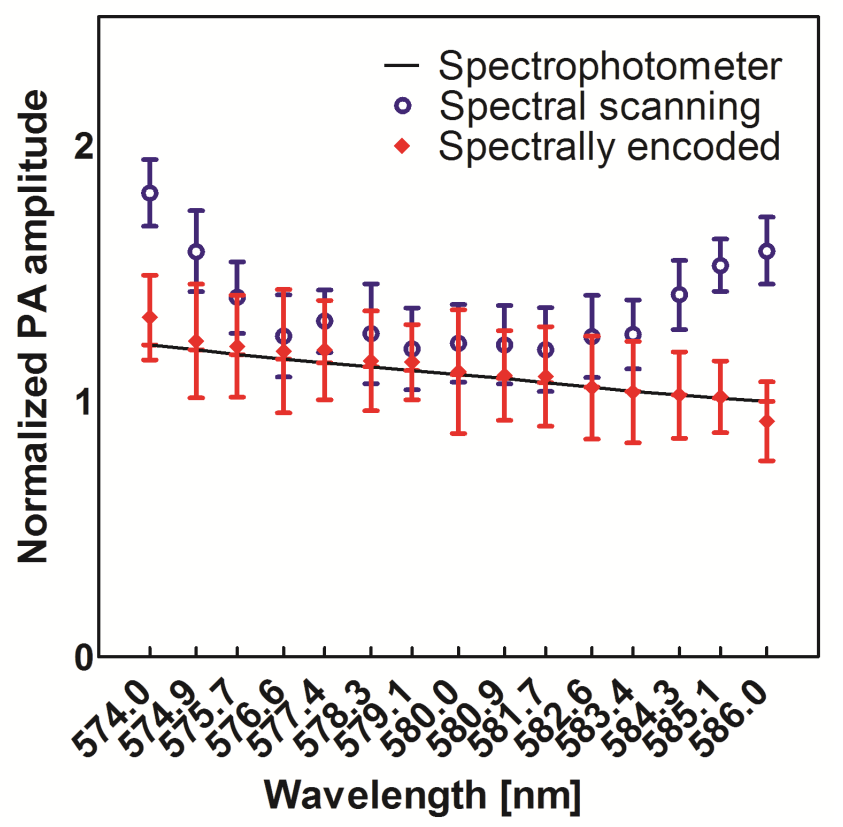

Figure 3. Red ink absorption spectrum. 


\section{DISCUSSION}

In summary, it has been shown that, by means of wavelength multiplexing, a spectrally encoded PA imaging system can improve the accuracy of spectroscopic PA measurements. Due to the multiplexing advantage, a broadband light source can be used advantageously. Future work will focus on exploring the supercontinuum fiber laser, which has a wider visible and NIR wavelength range for spectroscopic applications.

\section{REFERENCES}

[1] L. V. Wang, "Multiscale photoacoustic microscopy and computed tomography," Nature Photonics 3 (9), 503-509 (2009).

[2] D. Razansky, M. Distel, C. Vinegoni, R. Ma, N. Perrimon, R. W. Koster, and V. Ntziachristos, "Multispectral optoacoustic tomography of deep-seated fluorescent proteins in vivo," Nature Photonics 3, $412-417$ (2009).

[3] S. Hu, and L. V. Wang, "Photoacoustic imaging and characterization of the microvasculature," Journal of Biomedical Optics 15 (2010).

[4] K. H. Song, C. Kim, K. Maslov, and L. V. Wang, "Noninvasive in vivo spectroscopic nanorod-contrast photoacoustic mapping of sentinel lymph nodes," Eur J Radiol 70, 227-231 (2009).

[5] R. Ma, A. Taruttis, V. Ntziachristos, and D. Razansky, "Multispectral optoacoustic tomography (MSOT) scanner for whole-body small animal imaging," Opt Express 17, 21414-21426 (2009).

[6] J. P. Rice, S. W. Brown, J. E. Neira, and R. R. Bousquet, "A hyperspectral image projector for hyperspectral imagers," in Algorithms and Technologies for Multispectral, Hyperspectral, and Ultraspectral Imagery XIII(SPIE, Orlando, FL, USA, 2007), pp. 65650C-65612.

[7] Y. N. Billeh, M. Liu, and T. Buma, "Spectroscopic photoacoustic microscopy using a photonic crystal fiber supercontinuum source," Opt Express 18, 18519-18524.

[8] Thomas J. Allen and Paul C. Beard, "Dual wavelength laser diode excitation source for 2D photoacoustic imaging," Proc. SPIE 6437, 64371U (2007).

[9] Y. Wang, S. Hu, K. Maslov, Y. Zhang, Y. Xia, and L. V. Wang, "In vivo integrated photoacoustic and confocal microscopy of hemoglobin oxygen saturation and oxygen partial pressure," Opt Lett 36, 1029-1031.

[10] C. Zhang, K. Maslov and L. V. Wang, "Subwavelength-resolution label-free photoacoustic microscopy of optical absorption in vivo", Optics Letters 35, 3195-3197 (Oct. 2010).

[11] Y. Wang, K. Maslov, Y. Zhang, S. Hu, L. Yang, Y. Xia, J. Liu, and L. V. Wang, "Fiber-laser-based photoacoustic microscopy and melanoma cell detection," Journal Biomedical Optics 16, 011014 (2011).

[12] M. Harwit and N. Sloan, Hadamard transform optics. New York, USA: Academic press, 1979.

[13] M. Harwit, "Hadamard Transform Techniques in Spectroscopy and Imaging Spectroscopy," Infrared Physics 17, 457-457 (1977). 